\title{
ESTABILIZAÇÃO DE SISTEMAS FUZZY T-S INCERTOS USANDO REALIMENTAÇÃO DERIVATIVA
}

\author{
Emerson Ravazzi Pires da Silva* \\ e.ravazzi@bol.com.br \\ Marcelo C. M. Teixeira* \\ marcelo@dee.feis.unesp.br
}

\author{
Edvaldo Assunção* \\ edvaldo@dee.feis.unesp.br \\ Flávio Andrade Faria* \\ flaviof15eyahoo.com.br
}

\author{
${ }^{*}$ Faculdade de Engenharia \\ UNESP - Univ Estadual Paulista, Campus de Ilha Solteira \\ Departamento de Engenharia Elétrica, Laboratório de Pesquisa em Controle \\ Avenida José Carlos Rossi, n 1370, 15385-000 - Ilha Solteira, SP, Brasil
}

\begin{abstract}
Stabilization of uncertain T-S fuzzy systems using statederivative feedback

In some practical problems, for instance, in the suppression of vibration in mechanical systems, the state-derivative signals are easier to obtain than the state signals. Thus, a method for state-derivative feedback design applied to uncertain nonlinear systems is proposed in this work. The nonlinear systems are represented by Takagi-Sugeno fuzzy models during the modeling of the problem, allowing to use Linear Matrix Inequalities (LMIs) in the controller design. This type of modeling ease the control design, because, LMIs are easily solved using convex programming technicals. The control design aimed at system stabilisation, with or without bounds on decay rate. The efficiency of design procedure is illustrated through a numerical example.
\end{abstract}

KEYWORDS: State-Derivative Feedback, Fuzzy T-S Models, Parametric Uncertainties, Linear Matrix Inequalities (LMIs).

\section{RESUMO}

Em alguns problemas práticos, por exemplo, no controle de

Artigo submetido em 10/02/2010 (Id.: 01101)

Revisado em 08/05/2010, 11/09/2010, 27/02/2011, 06/04/2011

Aceito sob recomendação do Editor Associado Prof. Luis Fernando Alves Pereira vibrações de sistemas mecânicos, é mais fácil obter o sinal da derivada dos estados que o sinal dos estados. Assim, neste trabalho é proposto um método para o projeto da realimentação derivativa aplicada à sistemas não-lineares incertos. Durante a modelagem do problema os sistemas não-lineares são representados por modelos fuzzy TakagiSugeno, permitindo que o projeto do controlador seja realizado com Desigualdades Matriciais Lineares (em inglês, Linear Matrix Inequalities (LMIs)). Este tipo de modelagem facilita o projeto de controle, pois, LMIs são facilmente resolvidas utilizando técnicas de programação convexa. O projeto de controle visa a estabilização do sistema, com ou sem restrições na taxa de decaimento. A eficiência do método é ilustrada através de um exemplo numérico.

PALAVRAS-CHAVE: Realimentação Derivativa, Modelos Fuzzy T-S, Incertezas Paramétricas , Desigualdades Matriciais Lineares (LMIs).

\section{INTRODUÇÃO}

Devido a sua complexidade, analisar o comportamento dinâmico de sistemas não-lineares é uma tarefa muito difícil. Uma maneira de facilitar a análise é representar os sistemas não-lineares através de modelos Fuzzy Takagi-Sugeno (TS) (Takagi and Sugeno, 1985; Teixeira and Assunção, 2007; Teixeira et al., 2000). Esse tipo de modelagem consiste na representação de um sistema não-linear como uma 
combinação fuzzy de sistemas lineares (modelos locais), que descrevem o comportamento do sistema não-linear em diferentes pontos do espaço de estados. Dependendo da escolha dos modelos locais e dos parâmetros da combinação fuzzy (conhecidos como, funções de pertinência) o modelo fuzzy T-S pode fornecer uma representação aproximada (Teixeira and Żak, 1999) ou exata (Tanaka and Wang, 2001) do sistema não-linear. Nos últimos anos, houve um crescente interesse em pesquisas e aplicações de sistemas fuzzy (Barra Junior et al., 2005; Arrifano et al., 2007). A análise de estabilidade e o projeto de controladores são os conceitos mais importantes em sistemas fuzzy e, em alguns casos, são desenvolvidos empregando LMIs. A solução de problemas de otimização com restrições descritas por LMIs pode ser realizada com softwares de programação semi-definida, tais como o pacote "LMI control toolbox" do MATLAB ${ }^{\circledR}$ (Gahinet et al., 1995). Neste software, a solução ótima é encontrada usando algoritmos com tempo de convergência polinomial.

Normalmente, as condições de estabilidade são obtidas empregando o método direto de Lyapunov e a maioria dos trabalhos encontrados na literatura usam uma função quadrática de Lyapunov (do inglês, Common Quadratic Lyapunov Function (CQLF)) para garantir a estabilidade do sistema (Teixeira and Żak, 1999; Tanaka and Wang, 2001; Tanaka et al., 1998; Kim and Lee, 2000; Teixeira et al., 2003; Liu and Zhang, 2003; Teixeira et al., 2005; Teixeira et al., 2006; Fang et al., 2006; Andrea et al., 2008). Embora estes resultados sejam eficientes, já é conhecido na literatura que CQLFs conduzem a resultados conservadores. Consequentemente, alguns autores têm explorado o uso de funções de Lyapunov alternativas para obter condições de estabilidade menos conservadoras. Dentre as várias opções encontradas na literatura destacam-se: as funções de Lyapunov por partes (Arrifano et al., 2006), as funções de Lyapunov fuzzy (Mozelli et al., 2010) e as funções de Lyapunov polinomiais (Montagner et al., 2010). Apesar dos inúmeros esforços para a obtenção de resultados menos conservadores, todos os trabalhos mencionados até aqui consideram apenas a realimentação de estados ou realimentação da saída para o projeto do controlador e existem poucos resultados na literatura considerando a aplicação da realimentação derivativa em sistemas fuzzy T-S (Assunção, Faria, Teixeira and Cardim, 2007).

A motivação para o estudo da realimentação derivativa vem do fato que em sistemas que usam acelerômetros, é mais fácil obter os sinais da derivada dos estados do que os sinais dos estados. A partir da aceleração, é possível obter a velocidade com boa precisão, porém, é mais difícil obter o deslocamento (Abdelaziz and valášek, 2004). Logo, os sinais usados na realimentação são: a aceleração e a velocidade. Estes são as derivadas da velocidade e da posição que podem representar os estados do sistema. Devido a sua estrutura simples e ao baixo custo operacional, acelerômetros têm sido usados nas indústrias para a solução de vários tipos de problemas de engenharia (Abdelaziz and valášek, 2004; Abdelaziz and valášek, 2005; Reithmeier and Leitmann, 2003; Duan et al., 2005; Kwak et al., 2002b).

$\mathrm{Na}$ literatura especializada é possível encontrar vários métodos para a aplicação da realimentação derivativa em sistemas lineares (Kwak et al., 2002b; Reithmeier and Leitmann, 2003; Abdelaziz and valášek, 2004; Duan et al., 2005; Assunção, Teixeira, Faria, da Silva and Cardim, 2007; Faria, Assunção, Teixeira, Cardim and da Silva, 2009; Faria, Assunção and Teixeira, 2009). Porém, existem poucos resultados envolvendo sistemas não-lineares. Em (Assunção, Faria, Teixeira and Cardim, 2007) é apresentado um método para a $\mathcal{D}$-estabilização de sistemas Fuzzy T-S usando realimentação derivativa. Contudo, o método não pode ser aplicado em sistemas sujeitos a falhas estruturais.

Falhas estruturais são muito comuns em sistemas mecânicos e podem ser causadas pelo manuseio incorreto do equipamento, desgaste físico das peças, ou curto circuito em componentes eletrônicos. Falhas podem ser inseridas no projeto do controlador através de incertezas paramétricas, permitindo o uso de LMIs para a solução do problema (Isermann, 2006).

Usando realimentação derivativa, neste trabalho é proposto um método baseado em LMIs para a estabilização de sistemas não-lineares sujeitos a falhas. $\mathrm{O}$ resultados são obtidos empregando uma CQLF e durante o projeto do controlador também são consideradas restrições na taxa de decaimento. Do ponto de vista teórico, os resultados propostos são conservadores. Contudo, ainda não existem resultados na literatura empregando funções de Lyapunov alternativas para a estabilização de sistemas não-lineares, usando realimentação derivativa. O método apresentado em (Assunção, Faria, Teixeira and Cardim, 2007), também considera uma CQLF e não pode ser aplicado em sistemas sujeitos a falhas estruturais. A principal contribuição deste trabalho é propor uma extensão dos resultados apresentados em (Assunção, Teixeira, Faria, da Silva and Cardim, 2007; Faria, Assunção and Teixeira, 2009), para a estabilização de sistemas não-lineares. Ao longo do texto, os sistemas nãolineares são representados por modelos fuzzy T-S e as falhas estruturais são agregadas ao projeto através de incertezas paramétricas.

Neste trabalho é usada uma metodologia que separa as não-linearidades das incertezas paramétricas, resultando em condições menos conservadoras para o projeto do controlador (da Silva, 2009). Pois, tratando de sistemas que possuem diferentes complicações em sua estrutura, seria 
interessante fazer a distinção de ambas para que sejam tratadas de forma distinta, a fim de obter um resultado satisfatório e seguro, pois se não houver esta manipulação, os resultados alcançados serão de uma forma geral considerados conservadores (Arrifano et al., 2006).

A eficiência do método proposto é ilustrada através de um exemplo.

\section{FORMULAÇÃO DO PROBLEMA}

Qualquer sistema mecânico está sujeito a apresentar uma interrupção não desejada durante a sua operação. Denominamos este evento como "falha estrutural". Falhas estruturais podem ser inseridas no modelo de projeto através de incertezas paramétricas (Isermann, 2006). Desta forma, considere um sistema não-linear incerto descrito por:

$$
\dot{x}(t)=A(\alpha, \beta) x(t)+B(\alpha, \beta) u(t),
$$

sendo $A(\alpha, \beta) \in \mathbb{R}^{n \times n}$ e $B(\alpha, \beta) \in \mathbb{R}^{n \times m}$, funções matriciais contínuas que representam a dinâmica não-linear do sistema, tais que, as não-linearidades são representadas por $\alpha$ e as incertezas por $\beta, u(t) \in \mathbb{R}^{m}$ é a entrada de controle do sistema e $x(t) \in \mathbb{R}^{n}$ é o vetor de estados. O próximo passo, definido como "etapa de separação", é uma condição necessária para a aplicação do método proposto neste trabalho.

Hipótese 1. Suponha que o sistema (1) seja linear com relação as incertezas $\beta$, então o sistema pode ser reescrito como:

$$
\dot{x}(t)=(A(\alpha)+A(\beta)) x(t)+(B(\alpha)+B(\beta)) u(t),
$$

sendo que as matrizes $(A, B)(\alpha)$ são compostas somente pelos elementos não-lineares e as matrizes $(A, B)(\beta)$ somente pelos elementos incertos. Ou seja, as funções matriciais $(A, B)(\alpha, \beta)$ do sistema (1), podem ser decompostas em duas parcelas, separando as funções não-lineares das incertezas paramétricas.

Para efeito de esclarecimento, considere as matrizes $(A, B)(\alpha, \beta)$, descritas abaixo:

$$
\begin{aligned}
& A(\alpha, \beta)=\left[\begin{array}{ccc}
a_{11}(\alpha) & 1 & 0 \\
a_{21}(\beta)+5 & a_{22}(\beta) & 1 \\
a_{31}(\alpha, \beta) & a_{32}(\beta) & a_{33}(\alpha)
\end{array}\right], \\
& B(\alpha, \beta)=\left[\begin{array}{c}
b_{11}(\alpha)+1 \\
2 \\
b_{31}(\alpha, \beta)
\end{array}\right],
\end{aligned}
$$

realizando a separação das não-linearidades e das incertezas nas matrizes $(A, B)(\alpha, \beta)$, obtemos as seguintes matrizes
$(A, B)(\alpha)$ e $(A, B)(\beta)$

$$
\begin{gathered}
A(\alpha, \beta)=\underbrace{\left[\begin{array}{ccc}
a_{11}(\alpha) & 0,5 & 0 \\
2 & 0 & 0,2 \\
\bar{a}_{31}(\alpha) & 0 & a_{33}(\alpha)
\end{array}\right]}_{A(\alpha)} \\
+\underbrace{\left[\begin{array}{ccc}
0,5 & 0 \\
a_{21}(\beta)+3 & a_{22}(\beta) & 0,8 \\
\tilde{a}_{31}(\beta) & a_{32}(\beta) & 0
\end{array}\right]}_{A(\beta)},
\end{gathered}
$$

sendo $a_{31}(\alpha, \beta)=\bar{a}_{31}(\alpha)+\tilde{a}_{31}(\beta)$,

$$
B(\alpha, \beta)=\underbrace{\left[\begin{array}{c}
b_{11}(\alpha)+0,4 \\
1,1 \\
\bar{b}_{31}(\alpha)
\end{array}\right]}_{B(\alpha)}+\underbrace{\left[\begin{array}{c}
0,6 \\
0,9 \\
\tilde{b}_{31}(\beta)
\end{array}\right]}_{B(\beta)},
$$

sendo $b_{31}(\alpha, \beta)=\bar{b}_{31}(\alpha)+\tilde{b}_{31}(\beta)$.

Observação 1 Durante a etapa de separação, os elementos constantes das matrizes $(A, B)(\alpha, \beta)$ podem ser alocados na matriz com não-linearidades $(A, B)(\alpha)$, na matriz de incertezas $(A, B)(\beta)$, ou alocados proporcionalmente nas duas matrizes. Ou seja, a alocação dos elementos constantes não afeta o resultado final e garante que uma única matriz $(A, B)(\alpha, \beta)$ pode ser representada de várias formas diferentes, nas matrizes $(A, B)(\alpha)$ e $(A, B)(\beta)$.

Ao longo do texto o projeto do controlador é realizado considerando a representação fuzzy T-S do sistema nãolinear (1). Modelos fuzzy T-S são descritos da seguinte maneira (Takagi and Sugeno, 1985):

$$
\dot{x}(t)=\sum_{i=1}^{r} \sum_{j=1}^{p} \alpha_{i} \beta_{j}\left(A_{\left(\alpha_{i}, \beta_{j}\right)} x(t)+B_{\left(\alpha_{i}, \beta_{j}\right)} u(t)\right),
$$

ou ainda,

$$
\begin{aligned}
& \dot{x}(t)=\left(\sum_{i=1}^{r} \alpha_{i} A_{\alpha_{i}}+\sum_{j=1}^{p} \beta_{j} A_{\beta_{j}}\right) x(t) \\
& +\left(\sum_{i=1}^{r} \alpha_{i} B_{\alpha_{i}}+\sum_{j=1}^{p} \beta_{j} B_{\beta_{j}}\right) u(t) .
\end{aligned}
$$

As matrizes $A_{\alpha_{i}} \in \mathbb{R}^{n \times n}$ e $B_{\alpha_{i}} \in \mathbb{R}^{n \times m}$ são os parâmetros dos modelos locais e $\alpha_{i}$ suas respectivas funções de pertinência. As funções de pertinência satisfazem a seguinte relação:

$$
\left.\begin{array}{l}
(A, B)(\alpha)=\sum_{i=1}^{r} \alpha_{i}(A, B)_{\alpha_{i}}, \quad \alpha_{i} \geq 0, \\
\begin{array}{l}
i=1,2, \ldots, r, \\
\alpha=\left[\begin{array}{llll}
\alpha_{1} & \alpha_{2} & \ldots & \alpha_{r}
\end{array}\right]^{T} .
\end{array}
\end{array}\right\} \begin{aligned}
& \text { modelos } \\
& \text { fuzzy } T S \\
& \text { com } \alpha_{i} \\
& \text { conhecido }
\end{aligned}
$$


As matrizes $A_{\beta_{j}} \in \mathbb{R}^{n \times n}$ e $B_{\beta_{j}} \in \mathbb{R}^{n \times m}$ são os vértices do sistema incerto e os parâmetros $\beta_{j}$ são números reais desconhecidos, satisfazendo a relação:

$$
\left.\begin{array}{l}
(A, B)(\beta)=\sum_{j=1}^{p} \beta_{j}(A, B)_{\beta_{j}}, \quad \beta_{j} \geq 0, \\
j=1,2, \ldots, p, \quad \sum_{j=1}^{p} \beta_{j}=1, \\
\beta=\left[\begin{array}{llll}
\beta_{1} & \beta_{2} & \ldots & \beta_{p}
\end{array}\right]^{T} .
\end{array}\right\} \begin{aligned}
& \text { modelos de } \\
& \text { incertezas } \\
& \text { com } \beta_{j} \\
& \text { desconhecido }
\end{aligned}
$$

Como foi mencionado anteriormente, a precisão com que o sistema (7) aproxima o sistema (1) está diretamente relacionada com a escolha dos modelos locais e das funções de pertinência. Quando os estados do sistema (1) podem ser medidos em tempo real e são limitados, o método sistemático apresentado em (Tanaka and Wang, 2001) é capaz de gerar uma representação fuzzy exata do sistema não-linear. Neste caso, as funções de pertinência dependem de um ou mais estados e o sistema (7) representa um sistema quasi linear com parâmetros variaveis (Baranyi, 2009). Porém, nem sempre é possível medir os estados em tempo real, neste caso pode-se considerar funções de pertinência mais simples para a representação fuzzy. Pode-se, por exemplo, usar funções dependentes apenas do tempo e obter uma aproximação do sistema (1) usando sistemas lineares variantes no tempo.

\section{PROJETO DE CONTROLADORES USANDO REALIMENTAÇÃO DERIVATIVA}

O objetivo desta seção é encontrar condições suficientes para a existência de um ganho constante $K \in \mathbb{R}^{m \times n}$, tal que, ao realimentar o sistema (7) com a entrada:

$$
u(t)=-K \dot{x}(t)
$$

a matriz $(I+B(\alpha, \beta) K)$ seja inversível e o sistema em malha fechada seja quadraticamente estabilizável. Desta forma, substituindo (10) em (7) o sistema em malha fechada pode ser representado por:

$$
\dot{x}(t)=\underbrace{(I+B(\alpha, \beta) K)^{-1} A(\alpha, \beta)}_{A_{d}} x(t) .
$$

Observação 2 A inversibilidade da matriz $(I+$ $B(\alpha, \beta) K)$ é uma condição necessária para a aplicação da realimentação derivativa em sistemas não-lineares (Assunção, Teixeira, Faria, da Silva and Cardim, 2007).

A análise de estabilidade quadrática do sistema (11) pode ser realizada verificando as condições de existência de uma matriz simétrica $P \in \mathbb{R}^{n \times n}$, satisfazendo as condições de Lyapunov (Boyd et al., 1994):

$$
A_{d}^{T} P+P A_{d}<0, \quad P>0
$$

Assim, o problema da análise de estabilidade pode ser reduzido ao estudo de factibilidade das LMIs (12). Usando esse resultado, o próximo teorema encontra condições suficientes para a estabilidade quadrática do sistema (11). Durante a demonstração do teorema é usada a seguinte propriedade.

Propriedade 1 Para toda matriz $M$ não simétrica $M \neq$ $M^{T}$, se $M+M^{T}<0$, então $M$ é inversível (Boyd et al., 1994).

Teorema 1 Se existir uma matriz simétrica $X \in \mathbb{R}^{n \times n} e$ uma matriz $M \in \mathbb{R}^{m \times n}$ satisfazendo as LMIs,

$$
\begin{gathered}
X A_{\alpha_{i}}^{T}+A_{\alpha_{i}} X+X A_{\beta_{j}}^{T}+A_{\beta_{j}} X \\
+B_{\alpha_{l}} M A_{\alpha_{i}}^{T}+A_{\alpha_{i}} M^{T} B_{\alpha_{l}}^{T}+B_{\alpha_{l}} M A_{\beta_{j}}^{T} \\
+A_{\beta_{j}} M^{T} B_{\alpha_{l}}^{T}+B_{\beta_{j}} M A_{\alpha_{i}}^{T} \\
+A_{\alpha_{i}} M^{T} B_{\beta_{j}}^{T}+B_{\beta_{k}} M A_{\beta_{j}}^{T}+A_{\beta_{j}} M^{T} B_{\beta_{k}}^{T}<0 \\
X>0
\end{gathered}
$$

sendo, $i=1,2, \ldots, r$ e $j=1,2, \ldots, p$. Então, o sistema (11) é estabilizável, e um ganho que resolve o problema pode ser dado por

$$
K=M X^{-1}
$$

Prova: Supondo que (13) e (14) são factíveis e multiplicando ambos os lados de (13) por $\left(\alpha_{i} \times \alpha_{l} \times \beta_{j} \times \beta_{k}\right)$, segue de (8) e (9) que

$$
\begin{aligned}
X \sum_{i=1}^{r} & \alpha_{i} A_{\alpha_{i}}^{T}+\sum_{i=1}^{r} \alpha_{i} A_{\alpha_{i}} X+X \sum_{j=1}^{p} \beta_{j} A_{\beta_{j}}^{T}+\sum_{j=1}^{p} \beta_{j} A_{\beta_{j}} X \\
& +\sum_{l=1}^{r} \alpha_{l} B_{\alpha_{l}} M \sum_{i=1}^{r} \alpha_{i} A_{\alpha_{i}}^{T}+\sum_{i=1}^{r} \alpha_{i} A_{\alpha_{i}} M^{T} \sum_{l=1}^{r} \alpha_{l} B_{\alpha_{l}}^{T} \\
& +\sum_{l=1}^{r} \alpha_{l} B_{\alpha_{l}} M \sum_{j=1}^{p} \beta_{j} A_{\beta_{j}}^{T}+\sum_{j=1}^{p} \beta_{j} A_{\beta_{j}} M^{T} \sum_{l=1}^{r} \alpha_{l} B_{\alpha_{l}}^{T} \\
& +\sum_{j=1}^{p} \beta_{j} B_{\beta_{j}} M \sum_{i=1}^{r} \alpha_{i} A_{\alpha_{i}}^{T}+\sum_{i=1}^{r} \alpha_{i} A_{\alpha_{i}} M^{T} \sum_{j=1}^{p} \beta_{j} B_{\beta_{j}}^{T} \\
& +\sum_{k=1}^{p} \beta_{k} B_{\beta_{k}} M \sum_{j=1}^{p} \beta_{j} A_{\beta_{j}}^{T}+\sum_{j=1}^{p} \beta_{j} A_{\beta_{j}} M^{T} \sum_{k=1}^{p} \beta_{k} B_{\beta_{k}}^{T}<0 .
\end{aligned}
$$


Agrupando os termos, tem-se que:

$$
\begin{aligned}
& X\left(\sum_{i=1}^{r} \alpha_{i} A_{\alpha_{i}}^{T}+\sum_{j=1}^{p} \beta_{j} A_{\beta_{j}}^{T}\right)+\left(\sum_{i=1}^{r} \alpha_{i} A_{\alpha_{i}}+\sum_{j=1}^{p} \beta_{j} A_{\beta_{j}}\right) X \\
& +\left(\sum_{l=1}^{r} \alpha_{l} B_{\alpha_{l}}+\sum_{j=1}^{p} \beta_{j} B_{\beta_{j}}\right) M\left(\sum_{i=1}^{r} \alpha_{i} A_{\alpha_{i}}^{T}+\sum_{j=1}^{p} \beta_{j} A_{\beta_{j}}^{T}\right) \\
& +\left(\sum_{i=1}^{r} \alpha_{i} A_{\alpha_{i}}+\sum_{j=1}^{p} \beta_{j} A_{\beta_{j}}\right) M^{T}\left(\sum_{l=1}^{r} \alpha_{l} B_{\alpha_{l}}^{T}+\sum_{j=1}^{p} \beta_{j} B_{\beta_{j}}^{T}\right)<0,
\end{aligned}
$$

Pelo método da separação, sabe-se que:

$$
\begin{aligned}
& A(\alpha, \beta)=\left(\sum_{i=1}^{r} \alpha_{i} A_{\alpha_{i}}+\sum_{j=1}^{p} \beta_{j} A_{\beta_{j}}\right) \\
& B(\alpha, \beta)=\left(\sum_{i=1}^{r} \alpha_{i} B_{\alpha_{i}}+\sum_{j=1}^{p} \beta_{j} B_{\beta_{j}}\right),
\end{aligned}
$$

então de (17), chega-se em:

$$
\begin{aligned}
& X[A(\alpha, \beta)]^{T}+A(\alpha, \beta) X+B(\alpha, \beta) M[A(\alpha, \beta)]^{T} \\
& +A(\alpha, \beta) M^{T}[B(\alpha, \beta)]^{T}<0 .
\end{aligned}
$$

Substituindo $M=K X$ em (19) obtém-se,

$$
\begin{aligned}
& (I+B(\alpha, \beta) K) X[A(\alpha, \beta)]^{T} \\
& +A(\alpha, \beta) X\left(I+K^{T}[B(\alpha, \beta)]^{T}\right)<0 .
\end{aligned}
$$

Agora, usando a Propriedade 1 em (20) conclui-se que $(I+$ $B(\alpha, \beta) K) X[A(\alpha, \beta)]^{T}$ é inversível, logo $(I+B(\alpha, \beta) K)$ também é inversível. Considerando este fato, multiplique (20) à esquerda por $(I+B(\alpha, \beta) K)^{-1}$ e à direita por $\left(I+K^{T}[B(\alpha, \beta)]^{T}\right)^{-1}$ para obter:

$$
\begin{aligned}
& X[A(\alpha, \beta)]^{T}\left(I+K^{T}[B(\alpha, \beta)]^{T}\right)^{-1} \\
& +(I+B(\alpha, \beta) K)^{-1} A(\alpha, \beta) X<0 .
\end{aligned}
$$

Multiplicando (21) à direita e à esquerda por $P=X^{-1} \mathrm{e}$ considerando $A_{d}=(I+B(\alpha, \beta) K)^{-1} A(\alpha, \beta)$, chega-se na LMI:

$$
A_{d}^{T} P+P A_{d}<0,
$$

que é equivalente a condição (12) para o sistema (11). Portanto quando (13) e (14) são factíveis, segue de (21) que existe uma matriz simétrica $P=X^{-1}>0$, satisfazendo as condições de Lyapunov (12) para o sistema (11). Então, o ponto de equilíbrio $x=0$ do sistema é globalmente assintoticamente estável. E um ganho $K$ desejado pode ser obtido com (15).

Do ponto de vista de controle, a estabilidade do sistema nem sempre é suficiente, pois existem projetos que possuem restrições de desempenho. A modelagem em LMI permite que algumas restrições sejam adicionadas de maneira simples ao projeto do controlador. Por exemplo, restrições na taxa de decaimento.

\subsection{Estabilidade com restrição de taxa de decaimento}

Considere uma candidata a função de Lyapunov do tipo $V(x(t))=x(t)^{T} P x(t)>0$, com $\dot{V}(x(t))<0$ para todo $x(t) \neq 0$. A taxa de decaimento $\gamma, \gamma>0$, é garantida se a condição

$$
\dot{V}(x(t)) \leq-2 \gamma V(x(t)),
$$

for satisfeita para toda solução $x(t)$ do sistema (11) (Boyd et al., 1994). O próximo teorema encontra condições suficientes para que o sistema (11) tenha taxa de decaimento maior ou igual a $\gamma$.

Teorema 2 Dada uma constante real $\gamma>0$, se existir uma matriz simétrica $X \in \mathbb{R}^{n \times n}$ e uma matriz $M \in \mathbb{R}^{m \times n}$ satisfazendo as LMIs,

$$
\left[\begin{array}{cc}
E_{(1,1)} & X+B_{\alpha_{i}} M+B_{\beta_{j}} M \\
X+M^{T} B_{\alpha_{i}}^{T}+M^{T} B_{\beta_{j}}^{T} & -\frac{X}{2 \gamma}
\end{array}\right]<0,
$$

$$
X>0
$$

sendo, $i, l=1,2, \ldots, r, j, k=1,2, \ldots, p e$

$$
\begin{gathered}
E_{(1,1)}=X A_{\alpha_{i}}^{T}+A_{\alpha_{i}} X+X A_{\beta_{j}}^{T}+A_{\beta_{j}} X+B_{\alpha_{l}} M A_{\alpha_{i}}^{T} \\
+A_{\alpha_{i}} M^{T} B_{\alpha_{l}}^{T}+B_{\alpha_{l}} M A_{\beta_{j}}^{T}+A_{\beta_{j}} M^{T} B_{\alpha_{l}}^{T} \\
+B_{\beta_{j}} M A_{\alpha_{i}}^{T}+A_{\alpha_{i}} M^{T} B_{\beta_{j}}^{T}+B_{\beta_{k}} M A_{\beta_{j}}^{T}+A_{\beta_{j}} M^{T} B_{\beta_{k}}^{T} .
\end{gathered}
$$

Então, o sistema (11) é estabilizável com taxa de decaimento maior que $-\gamma$. E um ganho que resolve o problema pode ser dado por

$$
K=M X^{-1}
$$

Prova: Supondo que a LMI (23) é factível, e considerando o complemento de Schur (Boyd et al., 1994), tem-se que

$$
\begin{aligned}
& X A_{\alpha_{i}}^{T}+A_{\alpha_{i}} X+X A_{\beta_{j}}^{T}+A_{\beta_{j}} X+B_{\alpha_{l}} M A_{\alpha_{i}}^{T} \\
& +A_{\alpha_{i}} M^{T} B_{\alpha_{l}}^{T}+B_{\alpha_{l}} M A_{\beta_{j}}^{T}+A_{\beta_{j}} M^{T} B_{\alpha_{l}}^{T}+B_{\beta_{j}} M A_{\alpha_{i}}^{T} \\
& +A_{\alpha_{i}} M^{T} B_{\beta_{j}}^{T}+B_{\beta_{k}} M A_{\beta_{j}}^{T}+A_{\beta_{j}} M^{T} B_{\beta_{k}}^{T}<0 .
\end{aligned}
$$

Dessa maneira, segue da prova do Teorema 1 que a matriz $(I+B(\alpha, \beta) K)$ é inversível. Novamente, considerando o complemento de Schur (Boyd et al., 1994) em (23), segue que

$$
\begin{aligned}
& X A_{\alpha_{i}}^{T}+A_{\alpha_{i}} X+X A_{\beta_{j}}^{T}+A_{\beta_{j}} X+B_{\alpha_{l}} M A_{\alpha_{i}}^{T} \\
& +A_{\alpha_{i}} M^{T} B_{\alpha_{l}}^{T}+B_{\alpha_{l}} M A_{\beta_{j}}^{T}+A_{\beta_{j}} M^{T} B_{\alpha_{l}}^{T} \\
& +B_{\beta_{j}} M A_{\alpha_{i}}^{T}+A_{\alpha_{i}} M^{T} B_{\beta_{j}}^{T}+B_{\beta_{k}} M A_{\beta_{j}}^{T} \\
& +A_{\beta_{j}} M^{T} B_{\beta_{k}}^{T}+\left(X+B_{\alpha_{i}} M+B_{\beta_{j}} M\right) \\
& \times\left[2 \gamma X^{-1}\right]\left(X+B_{\alpha_{i}} M+B_{\beta_{j}} M\right)^{T}<0 .
\end{aligned}
$$


Considerando as mesmas operações realizadas na prova do Teorema 1 conclui-se que

$$
\begin{aligned}
& \quad(I+B(\alpha, \beta) K) X[A(\alpha, \beta)]^{T} \\
& \quad+A(\alpha, \beta) X\left(I+K^{T}[B(\alpha, \beta)]^{T}\right) \\
& +(I+B(\alpha, \beta) K)[2 \gamma X](I+B(\alpha, \beta) K)^{T}<0 .
\end{aligned}
$$

Agora, multiplicando (28) à esquerda por $P(I+B(\alpha, \beta) K)^{-1}$, à direita por $\left(I+K^{T}[B(\alpha, \beta)]^{T}\right)^{-1} P$ e considerando $P=X^{-1} \mathrm{e}$ $A_{d}=(I+B(\alpha, \beta) K)^{-1} A(\alpha, \beta)$, chega-se na LMI:

$$
A_{d}^{T} P+P A_{d}<-2 \gamma P,
$$

que é equivalente a condição (22) para o sistema (11). Portanto, quando as LMIs (23) e (24) são factíveis, o ponto de equilíbrio $x=0$ do sistema (11) é globalmente assintoticamente estável com taxa de decaimento maior que $\gamma$. E um controlador $K$ desejado pode ser obtido com (25).

A eficiência da metodologia proposta é ilustrada na solução de um exemplo numérico.

\subsection{Exemplos de simulação}

Para a solução numérica das LMIs foi usado o pacote "LMI control toolbox" presente no software MATLAB ${ }^{\circledR}$ (Gahinet et al., 1995). Durante a solução do exemplo são apresentados os ganhos obtidos com os Teoremas 1 e 2 .

\subsection{Sistema de Suspensão Ativa de um Veículo}

Considere o sistema de suspensão ativa dado na Figura 1.

Cujo modelo dinâmico pode ser descrito por (Reithmeier and Leitmann, 2003):

$$
\begin{array}{r}
{\left[\begin{array}{l}
\dot{x}_{1}(t) \\
\dot{x}_{2}(t) \\
\dot{x}_{3}(t) \\
\dot{x}_{4}(t)
\end{array}\right]=\left[\begin{array}{cccc}
0 & 0 & 1 & 0 \\
0 & 0 & 0 & 1 \\
\frac{-k_{x}-k_{2}}{M_{c}} & \frac{k_{2}}{M_{c}} & \frac{-b_{1}-b_{2}}{M_{c}} & \frac{b_{2}}{M_{c}} \\
\frac{k_{2}}{m_{s}} & \frac{-k_{2}}{m_{s}} & \frac{b_{2}}{m_{s}} & \frac{-b_{2}}{m_{s}}
\end{array}\right]\left[\begin{array}{c}
x_{1}(t) \\
x_{2}(t) \\
x_{3}(t) \\
x_{4}(t)
\end{array}\right]} \\
\\
+\left[\begin{array}{cc}
0 & 0 \\
0 & 0 \\
\frac{1}{M_{c}} & \frac{-1}{M_{c}} \\
0 & \frac{1}{m_{s}}
\end{array}\right] u(t),
\end{array}
$$

$\mathrm{O}$ vetor de estados é definido por $x(t)=$ $\left[\begin{array}{llll}x_{1}(t) & x_{2}(t) & x_{3}(t) & x_{4}(t)\end{array}\right]^{T}$, sendo que $x_{3}(t)=\dot{x}_{1}(t)$ e $x_{4}(t)=\dot{x}_{2}(t)$.

O sistema consiste em um veículo de massa $M_{c}$, um assento e uma pessoa, cuja massa total é $m_{s}$. As vibrações causadas

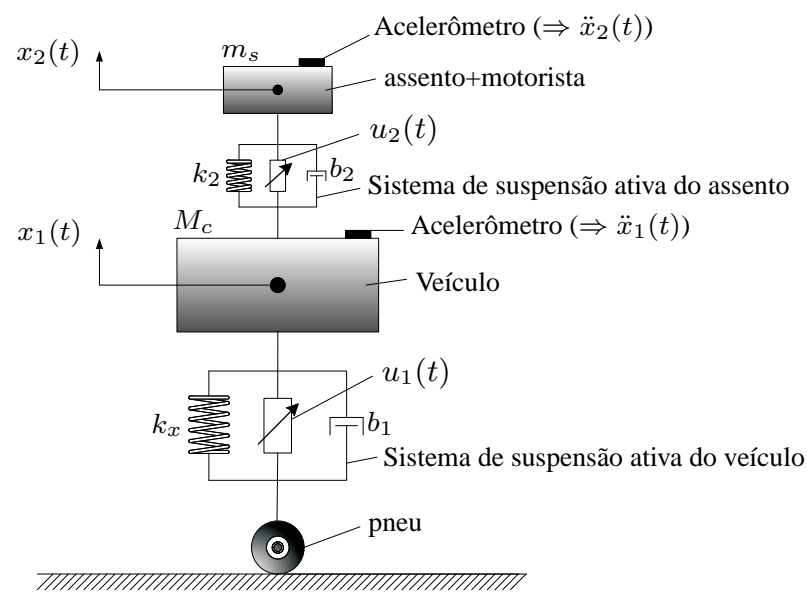

Figura 1: Sistema de suspensão ativa de um veículo.

por irregularidades na estrada podem ser atenuadas pelo sistema de suspensão do veículo (mola não-linear $k_{x}$ e amortecedor $b_{1}$ ). Mesmo assim, o motorista ainda pode sentir um pouco de vibrações. Uma maneira de melhorar o conforto do motorista é instalar um sistema de suspensão ativa no seu assento, composto por uma mola linear $k_{2}$ e um amortecedor $b_{2}$. Desta forma, pode-se diminuir as vibrações entre o motorista $\left(m_{s}\right)$ e o chassi do veículo $\left(M_{c}\right)$, modificando as entradas de controle $u_{1}(t)$ e $u_{2}(t)$ (veja Figura 1).

Considere $M_{c}=1500 \mathrm{~kg}, m_{s}=90 \mathrm{~kg}$ (assento $(20 \mathrm{~kg})+$ motorista $(70 \mathrm{~kg})), b_{2}=500 \mathrm{Ns} / \mathrm{m}$ e $k_{2}=5 \times 10^{3} \mathrm{~N} / \mathrm{m}$. Suponha também que o amortecedor do veículo esteja sujeito a uma falha estrutural, de tal forma que $b_{1}=2 \times 10^{3}$ enquanto o amortecedor está funcionando normalmente e $b_{1}=0$ após uma eventual quebra do amortecedor. $\mathrm{Ou}$ seja, o parâmetro $b_{1}$ é incerto e pertence ao intervalo $0 \leq$ $b_{1} \leq 2 \times 10^{3}(\mathrm{Ns} / \mathrm{m})$. O coeficiente não-linear da mola do veículo é dado por (Edwards and Penney, 2003): $k_{x}=$ $k_{1}\left(1+d^{2} x_{1}(t)^{2}\right)$, no qual $k_{1}=4 \times 10^{4} \mathrm{~N} / \mathrm{m}$ e $d=1$. Devido a restrições físicas (comprimento da mola), a variável de estado $x_{1}(t)$ é limitada no intervalo $-0,5 \leq x_{1}(t) \leq 0,5$ durante a operação do sistema.

Aplicando a etapa de separação no sistema (30), chega-se nas seguintes matrizes:

$$
A(\alpha)=\left[\begin{array}{cccc}
0 & 0 & 0,5 & 0 \\
0 & 0 & 0 & 0,5 \\
\tilde{f}_{31}(x(t)) & \frac{k_{2}}{2 M_{c}} & 0 & \frac{b_{2}}{2 M_{c}} \\
\frac{k_{2}}{2 m_{s}} & \frac{-k_{2}}{2 m_{s}} & \frac{b_{2}}{2 m_{s}} & \frac{-b_{2}}{2 m_{s}}
\end{array}\right]
$$


e

$$
\begin{gathered}
A(\beta)=\left[\begin{array}{cccc}
0 & 0 & 0,5 & 0 \\
0 & 0 & 0 & 0,5 \\
0 & \frac{k_{2}}{2 M_{c}} & \tilde{\mathrm{I}}_{33}(\beta) & \frac{b_{2}}{2 M_{c}} \\
\frac{k_{2}}{2 m_{s}} & \frac{-k_{2}}{2 m_{s}} & \frac{b_{2}}{2 m_{s}} & \frac{-b_{2}}{2 m_{s}}
\end{array}\right], \\
B(\alpha)=\left[\begin{array}{cc}
0 & 0 \\
0 & 0 \\
\frac{1}{2 M_{c}} & \frac{-1}{2 M_{c}} \\
0 & \frac{1}{2 m_{s}}
\end{array}\right] \text { e } B(\beta)=\left[\begin{array}{cc}
0 & 0 \\
0 & 0 \\
\frac{1}{2 M_{c}} & \frac{-1}{2 M_{c}} \\
0 & \frac{1}{2 m_{s}}
\end{array}\right],
\end{gathered}
$$

sendo $\tilde{f}_{31}(x(t))=\frac{-k_{x}-k_{2}}{M_{c}}$ a função que contém a nãolinearidade do sistema e $\tilde{\mathrm{I}}_{33}(\beta)=\frac{-b_{1}-b_{2}}{M_{c}}$ a função que contém a incerteza paramétrica (falha estrutural) do sistema. O sistema não-linear incerto (30) pode ser representado através de um modelo fuzzy T-S considerando o método de representação exata dado em (Taniguchi et al., 2001). Para tanto, considere a restrição $-0,5 \leq x_{1}(t) \leq 0,5$; Logo, $-36,6667 \leq \tilde{f}_{31}(x(t)) \leq-30$. Considerando este fato, o método apresentado em (Taniguchi et al., 2001) gera os seguintes modelos locais:

$$
\begin{aligned}
A_{\alpha_{1}}= & {\left[\begin{array}{cccc}
0 & 0 & 0,5 & 0 \\
0 & 0 & 0 & 0,5 \\
-30 & 1,6667 & 0 & 0,1667 \\
27,7778 & -27,7778 & 2,7778 & -2,7778
\end{array}\right], } \\
A_{\alpha_{2}}= & {\left[\begin{array}{cccc}
0 & 0 & 0,5 & 0 \\
0 & 0 & 0 & 0,5 \\
-36,6667 & 1,6667 & 0 & 0,1667 \\
27,7778 & -27,7778 & 2,7778 & -2,7778
\end{array}\right], }
\end{aligned}
$$$$
B_{\alpha_{1}}=B_{\alpha_{2}}=\left[\begin{array}{cc}
0 & 0 \\
0 & 0 \\
3,3333 \times 10^{-4} & -3,3333 \times 10^{-4} \\
0 & 5,5556 \times 10^{-3}
\end{array}\right] \text {. }
$$

Com as seguintes funções de pertinência:

$\alpha_{1}=\frac{\tilde{f}_{31}(x(t))+36,6667}{-30+36,6667}$ e $\alpha_{2}=1-\alpha_{1}$. Além disso, o parâmetro incerto $b_{1}$ gera os seguintes vértices para o politopo:

$$
\begin{aligned}
A_{\beta_{1}}= & {\left[\begin{array}{cccc}
0 & 0 & 0,5 & 0 \\
0 & 0 & 0 & 0,5 \\
0 & 1,6667 & -0,3333 & 0,1667 \\
27,7778 & -27,7778 & 2,7778 & -2,7778
\end{array}\right], } \\
A_{\beta_{2}}= & {\left[\begin{array}{cccc}
0 & 0 & 0,5 & 0 \\
0 & 0 & 0 & 0,5 \\
0 & 1,6667 & -1,6667 & 0,1667 \\
27,7778 & -27,7778 & 2,7778 & -2,7778
\end{array}\right], }
\end{aligned}
$$$$
B_{\beta_{1}}=B_{\beta_{2}}=\left[\begin{array}{cc}
0 & 0 \\
0 & 0 \\
3,3333 \times 10^{-4} & -3,3333 \times 10^{-4} \\
0 & 5,5556 \times 10^{-3}
\end{array}\right] \text {. }
$$

Assim, o modelo fuzzy T-S (7) obtido com os modelos locais e as funções de pertinência mencionados acima, geram uma representação exata do sistema não-linear (30).

Considerando a representação fuzzy T-S do sistema nãolinear (30), durante o projeto do controlador, as soluções encontradas pelo Teorema 1 foram:

$$
\begin{gathered}
P=X^{-1}=\left[\begin{array}{cccc}
0,077 & 0,000 & 0,020 & 0,000 \\
0,000 & 0,002 & 0,000 & 0,001 \\
0,020 & 0,000 & 0,008 & 0,000 \\
0,000 & 0,001 & 0,000 & 0,002
\end{array}\right], \\
M=10^{6} \times\left[\begin{array}{cccc}
0,205 & 0,327 & 1,434 & -0,061 \\
-0,001 & -0,333 & -0,174 & 3,342
\end{array}\right] .
\end{gathered}
$$

O ganho (15) obtido foi:

$$
K=M X^{-1}=10^{4} \times\left[\begin{array}{cccc}
4,445 & 0,066 & 1,619 & 0,015 \\
-0,347 & 0,213 & -0,148 & 0,712
\end{array}\right]
$$

Para a condição inicial $x(0)=\left[\begin{array}{llll}0,1 & 0,3 & 0 & 0\end{array}\right]^{T}, \mathrm{o}$ comportamento dinâmico do sistema mecânico (30), sem ação de controle $(u(t)=0)$, pode ser visto na Figura 2. Pela figura, o sistema mecânico (30) é naturalmente estável enquanto o amortecedor do veículo funciona normalmente $\left(b_{1}=2 \times 10^{3}\right)$. Contudo, após uma eventual falha no amortecedor o sistema passa a apresentar oscilações abruptas, gerando desconforto ao motorista e também risco de acidente. No entanto, ao considerarmos o ganho (31) na entrada de controle (10), o automóvel passa a ficar estável mesmo após a ocorrência de uma falha. Além disso, observando a Figura 3, nota-se que o comportamento do sistema controlado praticamente não muda, mesmo após a quebra do amortecedor $b_{1}$.
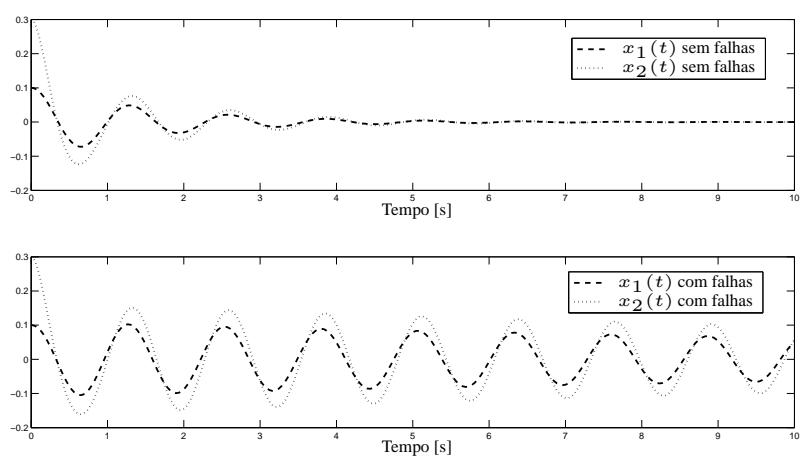

Figura 2: Simulação do sistema (30) sem entrada de controle.

Em ambos os casos, o sistema possui um tempo de duração do transitório em torno de $4 \mathrm{~s}$ para a suspensão do veículo e 

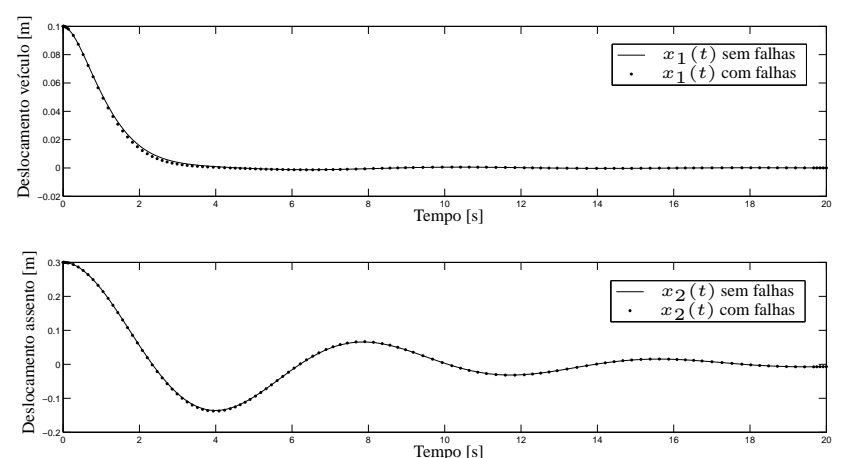

Figura 3: Sistema controlado com realimentação derivativa (Teorema 1).

20s para a suspensão do assento. Esta diferença do tempo de período transitório entre os estados $x_{1}(t)$ e $x_{2}(t)$ pode ocorrer devido às forças que atuam na suspensão ativa do veículo se dissiparem mais rápido do que as forças que atuam na suspensão ativa do assento.

A Figura 4 ilustra o esforço do sinal de controle necessário para garantir a convergência dos estados $x_{1}(t)$ e $x_{2}(t)$ para seus pontos de equilíbrio. Para o controle do veículo temos que $|u(t)|=3,6953 \times 10^{3} \mathrm{~N}$ (sem falhas) e $|u(t)|=$ $3,7226 \times 10^{3} \mathrm{~N}$ (com falhas). Para o assento, o esforço de controle $|u(t)|=0,9856 \times 10^{3} \mathrm{~N}$ (sem falhas) e $|u(t)|=0,9857 \times 10^{3} \mathrm{~N}$ (com falhas). Note que, mesmo após a ocorrência de falhas, o esforço do sinal de controle praticamente não se altera.
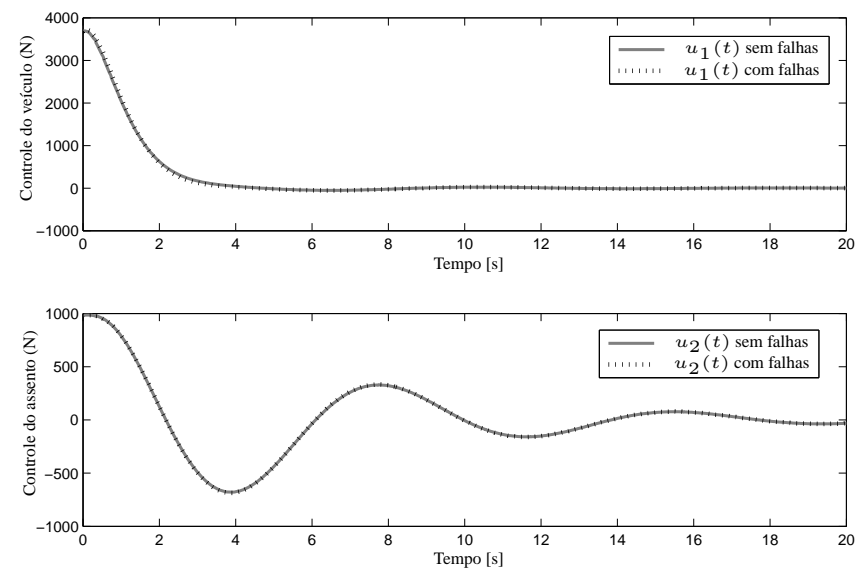

Figura 4: Esforço do sinal de controle considerando o Teorema 1.

Embora o Teorema 1 seja capaz de garantir a estabilidade do sistema, com ou sem falhas estruturais, o tempo de duração do transitório no banco aumentou, gerando mais desconforto ao motorista. Com o intuito de diminuir o tempo de duração do transitório, as LMIs do Teorema 2 foram resolvidas considerando $\gamma=2 \mathrm{e}$ as seguintes soluções foram encontradas:

$$
\begin{gathered}
P=X^{-1}=\left[\begin{array}{cccc}
0,946 & -0,057 & 0,046 & -0,005 \\
-0,057 & 0,099 & 0,029 & 0,008 \\
0,046 & 0,029 & 0,037 & 0,003 \\
-0,005 & 0,008 & 0,003 & 0,001
\end{array}\right], \\
M=10^{5} \times\left[\begin{array}{cccc}
0,318 & 0,560 & -0,681 & -1,365 \\
-0,008 & 0,333 & 0,204 & -4,574
\end{array}\right] .
\end{gathered}
$$

O ganho (25) obtido foi:

$$
K=M X^{-1}=10^{4} \times\left[\begin{array}{llll}
2,458 & 0,079 & 0,010 & -0,007 \\
0,076 & 0,052 & 0,031 & -0,007
\end{array}\right] .
$$

Utilizando a mesma condição inicial $x(0)$, o comportamento dinâmico do sistema realimentado, com e sem falha no amortecedor $b_{1}$, pode ser visto na Figura 5 .
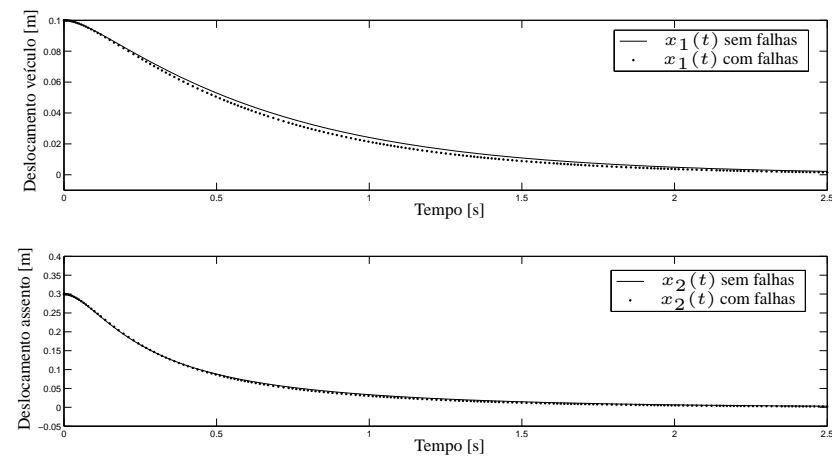

Figura 5: Sistema controlado com realimentação derivativa e restrição de taxa de decaimento (Teorema 2).

Note pela Figura 5 que, nos dois casos o sistema controlado possui tempo de período transitório em torno de $2 \mathrm{~s}$. Logo, o Teorema 2 foi capaz de melhorar o desempenho do sistema em relação à resposta do Teorema 1. A nova resposta é duas vezes mais rápida para a suspensão do veículo e dez vezes mais rápida para a suspensão do assento.

A Figura 6 ilustra o esforço de controle necessário que garante a convergência dos estados $x_{1}(t)$ e $x_{2}(t)$ ao equilíbrio considerando rapidez no sistema. Para o controle do veículo temos que $|u(t)|=3,3512 \times 10^{3} \mathrm{~N}$ (sem falhas) e $|u(t)|=3,5161 \times 10^{3} \mathrm{~N}$ (com falhas). Para o assento, o $\max$ esforço de controle $|u(t)|=0,5286 \times 10^{3} \mathrm{~N}$ (sem falhas) e $|u(t)|=0,5238 \times 10^{3} \mathrm{~N}$ (com falhas). Note que, mesmo $\max$ 
após a ocorrência de falhas, o esforço do sinal de controle praticamente não se altera.

Observe também que, o Teorema 2 projetou ganhos de realimentação melhorando a resposta transitória do sistema sem que houvesse aumento do esforço do sinal de controle. Ainda, para este exemplo, obtiveram-se valores de $|u(t)|$ menores que os valores obtidos pelo Teorema 1 .
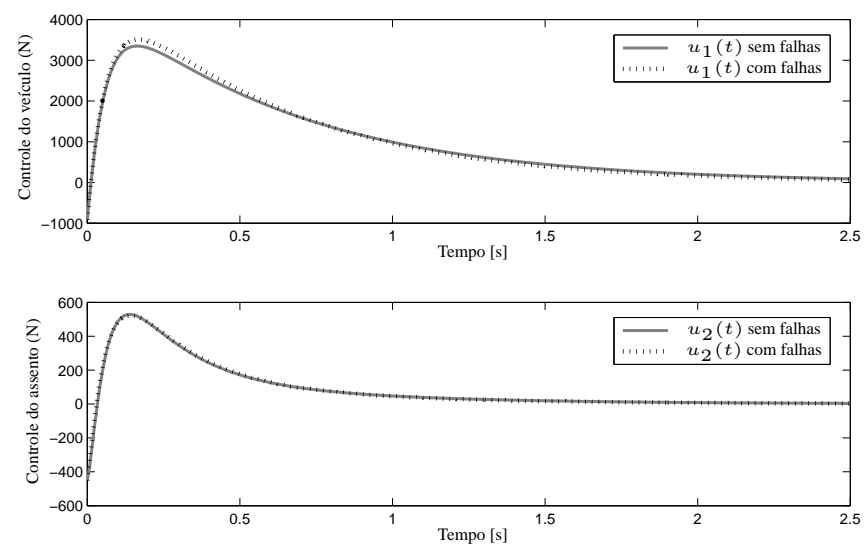

Figura 6: Esforço do sinal de controle considerando o Teorema 2.

\section{CONCLUSÕES}

Neste trabalho foi proposto um método para a estabilização de sistemas não-lineares incertos, usando realimentação derivativa. Os resultados são particularmente interessantes para a aplicação em sistemas mecânicos que utilizam acelerômetros como sensores. Durante a fase de projeto os sistemas não-lineares foram representados por modelos fuzzy T-S, permitindo o uso de LMIs para a solução do problema. Projetos de controle baseados em LMIs podem ser facilmente resolvidos em microcomputadores. Além disso, este tipo de modelagem facilita a adição de restrições de desempenho e de falhas estruturais no projeto do controlador. Neste trabalho também foi proposto uma metodologia para a abordagem de incertezas paramétricas em sistemas fuzzy T-S. A técnica consiste em analisar separadamente as não-linearidades das incertezas do sistema, resultando em condições menos conservadoras para o projeto do controlador. Ao contrário dos projetos com reguladores fuzzy, os resultados propostos neste trabalho utilizam ganhos fixos para o projeto do controlador. Do ponto de vista teórico estes resultados são mais conservadores, contudo eles dispensam a necessidade de avaliar as funções de pertinência em tempo real (Tanaka and Sugeno, 1992), facilitando a implementação prática do controlador. A eficiência dos métodos propostos é ilustrada na solução de um exemplo numérico.

Trabalhos futuros envolvem o projeto de controladores com funções de Lyapunov alternativas. Por exemplo, estender os resultados apresentados em da Silva et al. (2010) para a aplicação em sistemas não-lineares incertos.

\section{AGRADECIMENTOS}

Agradecemos à CAPES ${ }^{1}$, ao $\mathrm{CNPq}^{2}$ e a FAPESP ${ }^{3}$ pelo apoio financeiro a este trabalho.

\section{REFERÊNCIAS}

Abdelaziz, T. H. S. and valášek, M. (2004). Pole placement for SISO linear systems by state-derivative feedback, IEE Proceedings-Control Theory Applications 151(4): 377-385.

Abdelaziz, T. H. S. and valášek, M. (2005). State derivative feedback by LQR for linear time-invariant systems, 16th IFAC World Congress, Czech Republic.

Andrea, C. Q., Pinto, J. O. P., Assunção, E., Teixeira, M. C. M. and Junior, L. G. (2008). Controle ótimo $\mathcal{H}_{\infty}$ de sistemas não-lineares com modelos fuzzy TakagiSugeno, Sba: Controle \& Automação 19(3): 256-269.

Arrifano, N. S. D., Oliveira, V. A. and Cossi, L. V. (2006). Synthesis of an lmi-based fuzzy control system with guaranteed cost performance: A piecewise lyapunov approach, Sba: Controle \& Automação 17(2): 213-225.

Arrifano, N. S. D., Oliveira, V. A., Ramos, R. A., Bretas, N. G. and Oliveira, R. V. (2007). Fuzzy stabilization of power systems in a co-generation scheme subject to random abrupt variations of operating conditions, IEEE Transactions on Control Systems Technology 15(2): 384-393.

Assunção, E., Faria, F. A., Teixeira, M. C. M. and Cardim, R. (2007). Realimentação da derivada dos estados em sistemas fuzzy Takagi Sugeno, Anais do VIII Simpósio Brasileiro de Automação Inteligente, SBA, Florianópolis. 6p. (Artigo 29542).

Assunção, E., Teixeira, M. C. M., Faria, F. A., da Silva, N. A. P. and Cardim, R. (2007). Robust state-derivative feedback LMI-based designs for multivariable linear systems, International Journal of Control 80(8): 12601270 .

\footnotetext{
${ }^{1}$ Coordenação de Aperfeiçoamento de Pessoal de Nível Superior.

${ }^{2}$ Conselho Nacional de Desenvolvimento Científi co e Tecnológico.

${ }^{3}$ Fundação de Pesquisa do Estado de São Paulo.
} 
Baranyi, P. (2009). Convex hull generation methods for polytopic representations of LPV models, 7th International Symposium on Applied Machine Intelligence and Informatics, Herlany, Slovakia, pp. 69-74.

Barra Junior, W., Barreiros, J. A. L., da Costa Júnior, C. T. and Ferreira, A. M. D. (2005). Controle fuzzy aplicado à melhoria da estabilidade dinâmica em sistemas elétricos de potência, Sba: Controle \& Automação 16: 173-186.

Boyd, S., El Ghaoui, L., Feron, E. and Balakrishnan, V. (1994). Linear Matrix Inequalities in Systems and Control Theory, Volume 15 of Studies in Applied Mathematics, 2nd edn, SIAM Studies in Applied Mathematics, USA. http://www.stanford.edu/ boyd//mibook/.

da Silva, E. R. P. (2009). Controle robusto de sistemas não-lineares sujeitos a falhas estruturais, Master's thesis, Faculdade de Engenharia, Unversidade Estadual Paulista, Ilha Solteira.

da Silva, E. R. P., Assunção, E., Teixeira, M. C. M. and Faria, F. A. (2010). Condições relaxadas para o projeto de controladores de sistemas lineares incertos usando a realimentação da derivada dos estados, Anais do XVIII Congresso Brasileiro de Automática, SBA, Bonito, pp. 474-479.

Duan, Y. F., Ni, Y. Q. and Ko, J. M. (2005). State-Derivative feedback control of cable vibration using semiactive magnetorheological dampers, Computer-Aided Civil and Infrastructure Engineering 20(6): 431-449.

Edwards, C. H. and Penney, D. E. (2003). Elementary differential equations with boundary value problems, 5 edn, Prentice Hall, Englewood Cliffs.

Fang, C. H., Liu, Y. S., Kau, S. W., Hong, L. and Lee, C. H. (2006). A new LMI-based approach to relaxed quadratic stabilization of T-S fuzzy control systems, IEEE Transactions on Fuzzy Systems 14(3): 386-397.

Faria, F. A., Assunção, E. and Teixeira, M. C. M. (2009). Realimentação da derivada dos estados em sistemas multivariáveis lineares usando LMIs, Sba: Controle \& Automação 20(1): 83-93.

Faria, F. A., Assunção, E., Teixeira, M. C. M., Cardim, R. and da Silva, N. A. P. (2009). Robust state-derivative pole placement LMI-based designs for linear systems, International Journal of Control 82(1): 1-12.

Gahinet, P., Nemirovski, A., Laub, A. J. and Chilali, M. (1995). LMI control toolbox - For use with MATLAB, The Math Works Inc.
Isermann, R. (2006). Fault-Diagnosis systems: An introduction from fault detection to fault tolerance, Springer, Berlin.

Kim, E. and Lee, H. (2000). New approaches to relaxed quadratic stability condition of fuzzy control systems, IEEE Transactions on Fuzzy Systems 8(5): 523-534.

Kwak, S. K., Washington, G. and Yedavalli, R. K. (2002b). Acceleration feedback-based active and passive vibration control of landing gear components, Journal of Aerospace Engineering 15(1): 1-9.

Liu, X. and Zhang, Q. (2003). New approaches to $\mathcal{H}_{\infty}$ controller designs based on fuzzy observers for T-S fuzzy systems via LMI, Automatica 39(5): 1571-1582.

Montagner, V. F., Oliveira, R. C. L. F. and Peres, P. L. D. (2010). Relaxações convexas de convergência garantida para o projeto de controladores para sistemas nebulosos de Takagi-Sugeno, Sba: Controle \& Automação 21: 82-95.

Mozelli, L. A., Avellar, G. S. C., Palhares, R. M. and Santos, R. F. (2010). Alternative LMI conditions for TakagiSugeno systems via Fuzzy Lyapunov function, Sba: Controle \& Automação 21: 96-107.

Reithmeier, E. and Leitmann, G. (2003). Robust vibration control of dynamical systems based on the derivative of the state, Archive of Applied Mechanics 72(1112): $856-864$.

Takagi, T. and Sugeno, M. (1985). Fuzzy identification of systems and its applications to modeling and control, IEEE Transactions on Systems, Man, and Cybernetics 15(1): 116-132.

Tanaka, K., Ikeda, T. and Wang, H. O. (1998). Fuzzy regulators and fuzzy observers: Relaxed stability conditions and LMI-based designs, IEEE Transactions on Fuzzy Systems 6(2): 250-265.

Tanaka, K. and Sugeno, M. (1992). Stability analysis and design of fuzzy control systems, Fuzzy Sets and Systems 45(2): 135-156.

Tanaka, K. and Wang, H. (2001). Fuzzy control systems design and analysis: A linear matrix inequality approach, John Wiley and Sons, New York.

Taniguchi, T., Tanaka, K., Ohatake, H. and Wang, H. O. (2001). Model construction, rule reduction, and robust compensation for generalized form of Takagi-Sugeno fuzzy systems, IEEE Transactions on Fuzzy Systems 9(4): 525-537. 
Teixeira, M. C. M. and Assunção, E. (2007). Enciclopédia de Automática, Controle \& Automação, In: Luis Antonio Aguirre. (Org.), 1 edn, Edgard Blucher, São PauloSP, chapter Extensões para Sistemas Não-Lineares, pp. 218-243.

Teixeira, M. C. M., Assunção, E. and Avellar, R. G. (2003). On relaxed LMI-based designs for fuzzy regulators and fuzzy observers, IEEE Transactions on Fuzzy Systems 11(5): 613-623.

Teixeira, M. C. M., Catharino, M. F. R., Assunção, E. and Machado, E. R. M. D. (2005). A comparative study between two relaxed LMI-based fuzzy control designs, 2005 IEEE International Conference on Fuzzy Systems pp. 969-972.

Teixeira, M. C. M., da, Silva, N. A. P., Assunção, E. and Machado, E. R. M. D. (2006). Design of fuzzy regulators with optimal initial conditions compensation, 2006 IEEE International Conference on Fuzzy Systems pp. 84-91.

Teixeira, M. C. M., Pietrobom, H. C. and Assunção, E. (2000). Novos resultados sobre estabilidade e controle de sistemas não-lineares utilizando modelos fuzzy e LMI, Sba: Controle \& Automação 11(1): 37-48.

Teixeira, M. C. M. and Żak, S. H. (1999). Stabilizing controller design for uncertain nonlinear systems using fuzzy models, IEEE Transactions on Fuzzy Systems 7(2): 133-142. 